\title{
BMJ Open EQUIPT: protocol of a comparative effectiveness research study evaluating cross-context transferability of economic evidence on tobacco control
}

\author{
Subhash Pokhrel, ${ }^{1}$ Silvia Evers, ${ }^{2,3}$ Reiner Leidl, ${ }^{4}$ Marta Trapero-Bertran, ${ }^{5}$ \\ Zoltan Kalo, ${ }^{6}$ Hein de Vries, ${ }^{7}$ Andrea Crossfield, ${ }^{8}$ Fiona Andrews, ${ }^{9}$ Ailsa Rutter, ${ }^{10}$ \\ Kathryn Coyle, ${ }^{1}$ Adam Lester-George, ${ }^{11}$ Robert West, ${ }^{12,13}$ Lesley Owen, ${ }^{14}$ \\ Teresa Jones, ${ }^{1}$ Matthias Vogl, ${ }^{4}$ Cornel Radu-Loghin, ${ }^{15}$ Zoltan Voko, ${ }^{6}$ \\ Mirjana Huic, ${ }^{16}$ Doug Coyle ${ }^{1,17}$
}

To cite: Pokhrel S, Evers S, Leidl R, et al. EQUIPT: protocol of a comparative effectiveness research study evaluating cross-context transferability of economic evidence on tobacco control. BMJ Open 2014;4:e006945. doi:10.1136/bmjopen-2014006945

- Prepublication history for this paper is available online. To view these files please visit the journal online (http://dx.doi.org/10.1136/ bmjopen-2014-006945)

Received 17 October 2014 Accepted 31 October 2014

\section{CrossMark}

For numbered affiliations see end of article.

Correspondence to Dr Subhash Pokhrel; Subhash.Pokhrel@brunel. ac.uk

\section{ABSTRACT}

Introduction: Tobacco smoking claims 700000 lives every year in Europe and the cost of tobacco smoking in the EU is estimated between $€ 98$ and $€ 130$ billion annually; direct medical care costs and indirect costs such as workday losses each represent half of this amount. Policymakers all across Europe are in need of bespoke information on the economic and wider returns of investing in evidence-based tobacco control, including smoking cessation agendas. EQUIPT is designed to test the transferability of one such economic evidence base- - the English Tobacco Return on Investment (ROI) tool—to other EU member states.

Methods and analysis: EQUIPT is a multicentre, interdisciplinary comparative effectiveness research study in public health. The Tobacco ROI tool already developed in England by the National Institute for Health and Care Excellence (NICE) will be adapted to meet the needs of European decision-makers, following transferability criteria. Stakeholders' needs and intention to use ROI tools in sample countries (Germany, Hungary, Spain and the Netherlands) will be analysed through interviews and surveys and complemented by secondary analysis of the contextual and other factors. Informed by this contextual analysis, the next phase will develop country-specific ROI tools in sample countries using a mix of economic modelling and Visual Basic programming. The results from the country-specific ROI models will then be compared to derive policy proposals that are transferable to other EU states, from which a centralised web tool will be developed. This will then be made available to stakeholders to cater for different decision-making contexts across Europe.

Ethics and dissemination: The Brunel University Ethics Committee and relevant authorities in each of the participating countries approved the protocol. EQUIPT has a dedicated work package on dissemination, focusing on stakeholders' communication needs. Results will be disseminated via peer-reviewed publications, e-learning resources and policy briefs.

\section{Strengths and limitations of this study}

- EQUIPT is a rare multidisciplinary study designed to test the transferability of economic evidence around tobacco control.

- This study will provide evidence-based, practical and customisable decision-support tools to EU stakeholders.

- Unavailability of intervention effectiveness data on the full range of recommended activities and strategies may limit the scope of this research.

\section{INTRODUCTION}

Globally, tobacco smoking presents a significant problem, both in terms of the health of the population and the wider economic impact. No corner of the world is untouched by the effects of tobacco consumption and, in the EU alone, $28 \%$ of the population smokes, increasing to $29 \%$ in those aged 15-24 years. This results in considerable smoking-related socioeconomic inequalities across all EU member states, which translates to nearly 700000 premature deaths every year. ${ }^{1}$ Tobacco use is estimated to cost the European Community between $€ 98$ and $€ 130$ billion annually, with direct medical care costs and indirect costs (eg, workday losses) both representing half of this amount. These costs represented just above $1 \%$ of the EU Gross Domestic Product in 2000. Each EU resident (smoker or non-smoker) therefore bears a cost between $€ 211$ and $€ 281$ per year as the consequence of tobacco smoking in the region. ${ }^{2}$

The WHO Framework Convention on Tobacco Control (WHO FCTC) places a greater value on efforts that can help to reduce smoking prevalence and recommends 
that countries should implement evidence-based, comprehensive tobacco-control measures. ${ }^{3}$ The WHO FCTC has been ratified by all EU member states, and therefore efforts have been increasingly directed towards tackling tobacco smoking at all levels across the European Community through evidence-based interventions. ${ }^{4}$

A large, robust and consistent evidence base indicates that co-ordinated, high impact and comprehensive approaches are the most effective way to reduce smoking initiation, prevalence and intensity of consumption. ${ }^{56}$ However, policymakers and public health procurers often lack the data-and the financial justification-that can help them to make the case for comprehensive or other more focused investments to tackle the scourge of tobacco. In England, for example, the newly implemented Health and Social Care (2012) Act has placed a much greater role for Local Authorities in health prevention and, given the austere climate, this has resulted in more demand for information relating to the cost-effectiveness and return on investment (ROI) of tobacco control measures. Policymakers find it "difficult to present a business case for investing in public health interventions, due to a perceived lack of relevant data." ${ }^{77}$ This suggests that policymakers want to know the following two sets of questions: (1) If I were to invest money in providing several interventions now in the anticipation (based on current practice) that a certain number of smokers would eventually take up those interventions, what would be the extent of the return on my investment 'package' in the short, medium and long terms; and (2) What if I change the allocation of services (ie, shift the pattern of current uptake)?

To provide tobacco control policymakers and public health procurers with predictions of likely returns on investment, locally, regionally and nationally, arising from the funding of different tobacco control initiatives, Brunel University together with the National Institute of Health and Care Excellence (NICE) and regional tobacco control organisations in the UK have developed and tested a 'ROI' tool. ${ }^{8}$ The ROI tool is essentially a Markov model implemented in Microsoft Excel and is designed to provide measures of uncertainty around the ROI estimates via probabilistic sensitivity analysis. In an era of significant constraints on the public purse, this tool can contribute to making a powerful case for public health investments. The scarcity of relevant input data, however, poses a significant challenge to the adaptation of the tool to other countries. ${ }^{9-12}$

Most of the published evidence base on the costeffectiveness of cessation and tobacco control has been produced with no or minimal stakeholder engagement. Recent work by NICE in the UK suggested that for complex interventions or strategies, an individual economic metric-such as the incremental cost-effectiveness ratio (which shows how much extra cost decision-makers have to bear in order to gain one additional quality-adjusted life year)-is insufficient for decisionmakers to make an investment. ${ }^{7}$ The study found that there are a number of other economic metrics that are deemed important in policy decisions. Moreover, the study found that lots of different tools were being used to support purchasing decisions-most of which required judgements on the part of users. This was further corroborated by another study in which the authors consulted with potential stakeholders prior to developing their economic model. ${ }^{13}$ This strongly indicates the importance of engaging stakeholders in the evidence generation process in order to ensure the wider use of such evidence and to facilitate its transferability to other countries.

In this paper, we describe EQUIPT (European study on quantifying utility of investment in protection from tobacco), a comparative effectiveness research (CER) study evaluating the cross-context transferability of economic evidence on tobacco control.

\section{METHODS AND ANALYSIS Objective}

The overarching aim of EQUIPT is to provide healthcare policymakers in the EU with bespoke information about the potential economic and wider returns to be expected from investing in evidence-based tobacco control agendas. EQUIPT has the ultimate ambition of underpinning health authority decisions on the development and/or harmonisation of new strategies for tobacco control projects for health promotion and disease prevention in the EU by disseminating the ROI concept and tools across Europe. In the first instance, it will test the applicability and implementation of the already developed ROI tool in four other EU member states. Next, it will test the transferability of the ROI methods to guide comprehensive tobacco-control policies in other EU countries.

\section{Study design}

EQUIPT is a multicentre, interdisciplinary CER study in public health. The NICE Tobacco ROI tool-which is a practical, customisable economic model developed in England to help make real-world decisions in the context of local government decision-making-will be adapted to meet the needs of European decision-makers. Locating itself in the theory of diffusion of innovation ${ }^{14}$ and transferability of economic evidence, ${ }^{10}$ EQUIPT will co-create ROI tools to compare the effectiveness of tobacco control strategies both within and across several EU countries.

We incorporate the 'inverted cone' approach (figure 1) in which: (1) the tip represents the NICE ROI tool (ie, economic evidence); (2) the middle section represents the working space (ie, the extent to which this evidence can be applied in sample countries) and (3) the top level represents the extended benefit (ie, the extent to which the policy recommendations coming out from sample countries can be transferred to other out-of-sample EU countries). At the heart of this approach is the translational research framework, ${ }^{15} 16$ which allows us to utilise different quantitative and 
Figure 1 'Inverted Cone' design of EQUIPT for evidence transfer within the Translational Research Framework (ROI, Return on Investment).

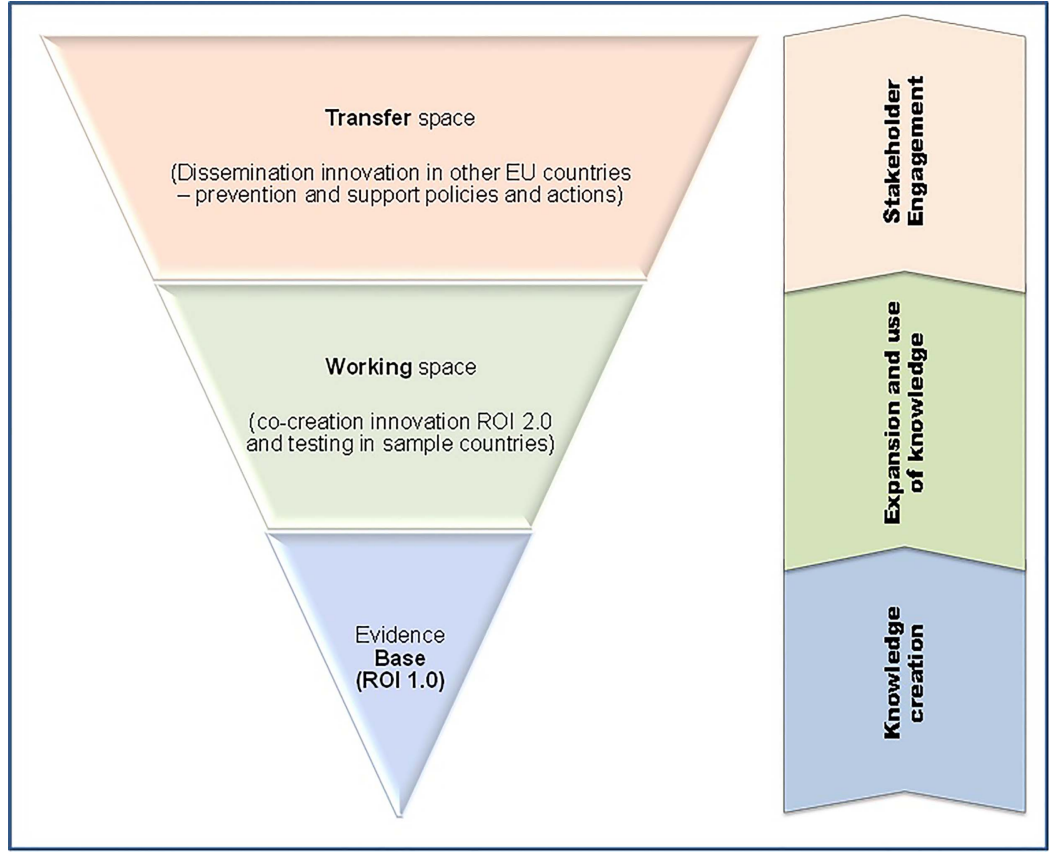

qualitative methods while benefiting from the experience and expertise of multidisciplinary consortium members.

\section{Study participants}

Four countries in Europe-Germany, Spain, Hungary and the Netherlands-are included as evidencereceiving countries (ie, sample countries). The choice of sample countries is deliberate and represents a wide array of potential transferability factors, which may then help to make statements for other European countries outside this sample. Geographically, this sample covers the whole continuum from West to East and North to South and includes a wide range of cultural, behavioural, economic and other issues which may be considered when transferring evidence from one country to another. It is anticipated that other countries not considered in EQUIPT are likely to show outcome-relevant characteristics which are similar to at least one of the sample countries.

\section{Collaborating institutions}

This study is funded by the Framework Seven Programme (FP7) of the European Community and co-ordinated by the Health Economics Research Group at Brunel University (UK). The collaborating institutions in the sample countries are: the Caphri School for Public Health and Primary Care at Maastricht University (the Netherlands), the Institute of Health Economics and Health Care Management at Helmholtz Zentrum München (Germany); the Syreon Research Institute (Hungary) and the Centre for Research in Health and Economics, Pompeu Fabra University (Spain). A wide range of institutions collaborate to provide multidisciplinary inputs required by the study: the National Institute for Health and Care Excellence (UK), LeLan Solutions (UK); the National Centre for Smoking
Cessation and Training (UK), the European Network for Smoking and Tobacco Prevention (Belgium); the Agency for Quality and Accreditation in Health Care and Social Welfare (Croatia) and the NHS Bristol Primary Care Trust (UK) on behalf of Smokefree South West, Tobacco Free Futures and FRESH North East.

\section{Interventions}

EQUIPT will consider the following two groups of interventions:

1. Smoking cessation interventions to include behavioural interventions, pharmacotherapy and mixed (behavioural+pharmacotherapy) implemented at the individual smoker level.

2. Tobacco control interventions to include smoking prevention and cessation interventions targeted at the population level.

The specific interventions to be included in the tool may vary from one sample country to the other and will be based on the following three criteria:

- Analysis of local stakeholders' views on the availability and relevance of interventions in a country.

- The interventions are supported by clear evidence on effectiveness and/or cost-effectiveness, defined by high-quality 'comparative' data coming from randomised trials, economic evaluations or real-world observational studies.

- The interventions are recommended already, or have potential for recommendation, by:

A. The country's Department of Health (DH) or Health Technology Assessment (HTA) body (if in existence);

B. Other (similar) EU country's DH or HTA;

C. NICE in the UK. 
A 'package' of interventions (rather than a single intervention) will be evaluated for its ROI. The 'package' can be the current practice (ie, mix of all existing interventions at their current level of uptake) or alternative practice (ie, mix of interventions customised to reflect the policymakers' needs, eg, by shifting current uptake or removing one or more less effective interventions).

\section{Comparators}

The comparators will be: (1) baseline, that is, none of the interventions in place; and (2) current practice, that is, the existing provision of services.

\section{Data and analysis}

Table 1 summarises the tasks, the data and collection method, and the analysis plan.

In tasks 1 and 2, we will define the contexts in which tobacco control sits in sample countries in order to inform the applicability and transferability of the ROI model to those countries. Desk reviews and stakeholder interviews will provide data that will help assess the (1) availability and relevance of different interventions in sample countries; (2) implications for attuning current ROI algorithms; (3) needs of local policymakers for including economic evidence in their decision-making and (4) factors that are crucial for ROI adoption in sample countries. We will collect both qualitative data (eg, a description of different types of cessation services and tobacco control interventions and views of policymakers) and quantitative data (eg, population size and composition (age/gender); smoking and ex-smoking prevalence; costs of interventions and quit rates; uptake rates of interventions; productivity impacts of smoking). The Integrated Change model ${ }^{17}$ will be used to study the factors influencing the stakeholders' intention to use ROI tools by assessing their awareness of ROI tools, motives for using such a tool, and future intention and action plans to use the ROI tools. Both qualitative (openended questionnaires and workshops) as well as quantitative methods will be applied. An assessment of preferred usability will also be included to enhance future adoption and implementation of ROI tools.

In task 3, we will adapt the current ROI model to reflect the needs of decision-makers in sample countries. This ROI model uses a Markov state transition model with three states: Smoker, Former Smoker and Death. $^{8}{ }^{18}$ At the start of the simulation, the entire cohort begins as smokers. With each 1 year cycle, the cohort is assigned a set of transition probabilities which allows them to either stay within their current state or move to one of the other two states. Death is an absorbing state, meaning that those who enter this state remain within the state. Within each cycle, both smokers and former smokers may develop smoking attributable diseases: lung cancer, coronary heart disease, myocardial infarction, chronic obstructive pulmonary disease or stroke. The model provides estimates of both short-term (reduced healthcare use by

Table 1 Task, type of data and analysis plan in EQUIPT

\begin{tabular}{lll}
\hline Task & Data/collection methods & Analysis \\
\hline 1. Comparative assessment of contexts in which & Desk reviews of literature (QL) & Narrative literature analysis \\
tobacco sits in sample countries & Survey data (QN) & Statistical analysis \\
& Model input parameters (QN) & Expert review of evidence
\end{tabular}

2. Needs assessment to inform how differential Stakeholder interviews (QL/QN) contexts demand the most appropriate approach to $\mathrm{ROI}$ tool development and usage of the tool

3. Development of core components and country-specific components (local models) of the ROI tool

4. CER, based on local ROI models, to identify key determinants of policy decisions

\section{Drawing policy recommendations}

6. Testing of transferable policy recommendations

7. Assessing the most-preferred method for communicating findings
Statistical analysis on the intention to use, complemented by content analysis

Stakeholder consensus meetings (QL) Ranking exercise and qualitative assessment

Economic modelling (QN)

Quality checks and validation (QL/QN) User-interface (QN)

Data based on the outcomes of probabilistic sensitivity analysis $\mathrm{ROI}$ values for each country

Stakeholder workshops to contextualisation of research findings (QL)

Additional data from out-of-sample countries (QN/QL)

Online survey (QL/QN) 
those stopping smoking, productivity gains, social care costs avoided due to reduced strokes and reduced events in both children and adults exposed to passive smoking) and long-term (smoking attributable deaths, life years, quality-adjusted life years (QALYs) and costs) outcomes.

In the Markov state transition model, the prevalence of smoking-related diseases for smokers and former smokers are estimated through assessing population attributable fractions. Costs and utilities are assigned to each disease. Utility decrements are associated not only with smoking attributable diseases but also as a result of being a smoker or a former smoker. Outcomes are assessed for different population cohorts based on age (18-85 years) and gender. To obtain population level estimates, these cohort level estimates are weighted by the percentage of the smoking population falling into each age and gender cohort. The model adopts a quasisocietal perspective in which costs and benefits to the healthcare system are incorporated in addition to productivity gains and reduced need for social care due to reduced smoking. Costs and outcomes are discounted at a rate of $3.5 \%$ per annum (although a discount rate between $0 \%$ and $10 \%$ can be chosen).

In designing the country-specific models in task 3 , we will follow standard guidelines on transferring economic models to other contexts. ${ }^{19-23}$ The data to populate these models will be obtained from published sources and through additional data analyses. Where available, country-specific data will be used in the local models, but we anticipate that some data (eg, intervention effectiveness) may have to be sourced from the best available evidence (eg, Cochrane review). A user-friendly interface will be developed using Visual Basic for Applications (VBA) as the front-end to ensure easy access to the MS Excel-based models. The models will be validated in line with best practice guidelines, using an adapted version of the available checklists. ${ }^{21} 2425$ Model outputs will cover a range of indicators (table 2), although the final indicators and data are to be determined by the outcomes of tasks 1 and 2.

In task 4, we will conduct a comparative analysis of the ROI in tobacco control using the outputs from countryspecific models to inform the transferability of results.
This analysis will focus on the burden of tobacco and the ROI of a 'comparable-in-content' package of tobacco control interventions across sample countries.

In tasks 5 and 6 , we will aim to facilitate the transfer of comparative effectiveness data to other countries in order to make timely and sensible policy recommendations, even in the absence of relevant evidence for the country of interest. This will be done by identifying key variability factors that, if collected for out-of-sample countries and used in the ROI model, could provide similar policy recommendations. Relevant parameters will be identified through importance analysis techniques which are quantitative approaches for estimating the impact of changes in input parameters on the output uncertainty. ${ }^{26}$ This will be tested by collecting those variables in a limited number of out-of-sample EU countries $(n=3)$ as well as feedback from a range of stakeholders via workshops. The selection of these countries will be based on the following criteria: that they are significantly different from the four sample countries in terms of stage of tobacco control, health status and use of HTA in decision-making and that there is a higher potential to save life years from tobacco control and quit-support programmes.

Tasks 4-6 will inform the creation of a web-based tool by combining information on core components and country-specific components to allow timely and sensible policy recommendations for decision-makers across Europe. The country-specific ROI models developed for sample countries will be redeveloped and reprogrammed into a unified, web-based tool that can be used by other EU countries to estimate the ROI of their tobacco control agenda. Therefore, a fully validated generic web-based tool will ultimately become the final deliverable product of this project.

In task 7 , we will assess the most preferred method for communicating study findings to ensure that they are used to devise tobacco control policies across Europe. This will be achieved by an online survey and will also be partly informed by the outcomes from task 1 .

\section{Stakeholder engagement}

Key stakeholders will be identified right from the beginning (table 3). Stakeholders will provide key inputs to all

Table 2 Examples of intended model outputs to aid decision-making

Costs of tobacco use

Avoidable Burden of Disease

Incremental Cost-Effectiveness Ratio or ICER

Benefit Cost Analyses or BCA

Net Present Value Analyses or NPV
The economic costs (in $€$ ) of tobacco use in the selected area, from both healthcare and wider perspectives

The number of quality-adjusted life years (QALYs) gained per 1000 population over 2, 5,10 years and a lifetime, as the result of implementing a package of interventions Healthcare costs per smoking related death averted, per life year gained and per QALY gained over $2,5,10$ years and a lifetime, as the result of implementing a package of interventions

Healthcare savings benefit-cost ratios, healthcare savings and value of health gains benefit-cost ratios, as the result of implementing a package of interventions Healthcare cost savings per smoker, healthcare cost savings and value of health gains per smoker, as the result of implementing a package of interventions 


\section{Method of engagement}

Interviews

\section{Engagement purpose}

To learn about the needs of local policymakers for including economic evidence in their decision-making and the factors that are crucial for return on investment (ROI) adoption in sample countries

Consensus workshops (to be held separately in each sample country)

Piloting of a country-specific ROI tool

Workshop on UK policy experience

Survey

To assess the project website using the I-change model

To make project information and results available to stakeholders

To reach out to policy, practice and research communities and to raise awareness and interest for the new ROI too ecommendations for the next phase of the project (adaption of ROI tool in sample countries)

To market test the relevance and applicability of the country-specific ROI tools

To understand the experience of users of $\mathrm{ROI}$ tools on its applicability, relevance and the extent to which helped in developing policy proposals at local and regional levels in England. This will inform and acilitate the research process and encourage policymaking

devise a set of policy proposals relevant to the out-of-sample countries for the implementation of evidence-based public health policies and support the validation of the ROI online tool translation into nationa/ local policy
International workshop implementation in their country

\section{Definition of stakeholder}

One or more of the following: health policymakers; purchasers of services; generators of evidence leading to policy/procurement/delivery of services advocates of health promotion; experts including clinicians and those specialising in tobacco control; financial authorities

Interviewed stakeholders

\section{Examples}

Two types of stakeholder groups will be targeted: (1) National and European stakeholders consisting of policymakers, academics, health authorities, insurance companies, advocacy groups, ministry of finance, national committees, clinicians and health technology assessment (HTA) professionals and (2) Experts on smoking cessation and HTA

Interviewed stakeholders

Interviewed stakeholders

Interviewed stakeholders

5 regional/local and 5 national UK stakeholders with previous experience in using the $\mathrm{ROI}$ tools

Directors of Public Health, service commissioners (procurers), local authority representatives (policy and strategy), tobacco control managers, advocacy group representatives

Stakeholders from those countries with limited capacity and budget for the implementation of evidence-based public health policy (mostly Central and Eastern European Countries)

Relevant stakeholders are those agencies that may wish to use HTA assessments when making decisions about which tobacco control strategies/ programmes they wish to recommend for

Those who are expected to use the website to access project information and results

Relevant stakeholders are those agencies that may wish to use HTA assessments when making decisions about which tobacco control strategies/ programmes they wish to recommend for implementation in their country
Central and Eastern European national representatives of: policymakers (from ministries of health, social welfare, finance and science and education); health professionals (from national public health institutes, national medical associations); researchers (from the area of public health service researchers); insurers/payers; patient organisations (condition/disease specific), HTA agencies

Officers from the ministry of health responsible for Tobacco Control, relevant health organisations (eg, national Tobacco Control agencies, Heart Societies, Cancer Societies, Asthma Societies, Regional Health Authorities, Health Insurance Companies, HTA groups)

Project partners; potential users of the ROI tools, media; lay public

Stakeholders thus include officers from the ministry of health responsible for Tobacco Control, relevan health organisations (eg, national Tobacco Control agencies, Heart Societies, Cancer Societies, Asthma Societies, Regional Health Authorities, Health Insurance Companies, HTA groups) 
stages of the research: needs identification (including the need for a user-friendly interface), piloting and testing of ROI concepts and tools in the local setting, drawing policy implications, testing transferability assumptions and creating effective dissemination plan.

\section{Ethics and dissemination}

The usual ethical issues-maintaining confidentiality, anonymity and data protection for primary data (eg, stakeholder interviews) and overall good research practice -apply to EQUIPT. As this research is led by Brunel University London, the Brunel University London Research Ethics Committee (UK) has reviewed this research and given full ethical clearance. The respective authorities in sample countries (Ethik-Kommission, Bayerische Landesärztekammer from Germany, Egészségügyi Tudományos Tanács, Tudományos És Kutatásetikai Bizottság from Hungary, and Parc de Salut MAR-Clinical Research Ethics Committee from Spain and Medischethische toetsingscommissie (METC) azM/UM from the Netherlands) have also provided clearance.

EQUIPT has a dedicated work package on dissemination of findings. The ROI tools will be available for public download through the project's website (http:// equipt.ensp.org) together with the accompanying User Guide, Technical Reports and worked-out examples. This will form the part of e-learning resources. The major analytical findings will be disseminated through peer-reviewed publications in scientific journals, presentations in conferences, policy briefs and media briefs.

\section{STATUS OF STUDY}

EQUIPT is a 3-year project that started on 1 October 2013 and will end on 30 September 2016.

\section{DISCUSSION}

EQUIPT is a rare multidisciplinary study designed to test the transferability of economic evidence around tobacco control and will provide evidence-based, practical and customisable ROI tools to actual decision-makers. The findings are expected to promote and disseminate the ROI methods and results to foster evidence-driven decision-making on comprehensive tobacco control across Europe.

The primary aim of transferring comparative effectiveness data to other countries is to make timely and sensible policy recommendations, even in the absence of relevant evidence for the country of interest. This is especially beneficial for countries with fewer analytical resources, where there is a lack of relevant input data to adapt the ROI model and in which there is a higher potential to save life years from tobacco control and quit support strategies. ${ }^{12}$ There is a limited understanding of the causes of variability in cost-effectiveness data, and this presents a key barrier to the transferability of the economic evaluation results. ${ }^{9}{ }^{27}$ Some authors suggest that "there is a lack of empirical studies which prevents stronger conclusions regarding which transferability factors are most important to consider and under which circumstances." 11 Nevertheless, the transfer of evidence to other countries may be possible if: (1) we identify those factors which cause the most variability in the relative success of tobacco control and quit-support strategies across countries and (2) the countries of interest are appropriately reflected in the existing data. ${ }^{10}$ Adapting an economic model may require an evaluation of those model components that are similar across countries (core components) and those that vary between countries (country-specific components). For example, the EUnetHTA programme "attempt[s] to define and standardise elements of an HTA" by dividing relevant information on the technology under assessment "into standardised pieces, each of which describing one or more aspects of the technology that is likely to be useful when considering the adoption or rejection of the technology." ${ }^{23}$ As described above, the EQUIPT study will follow a stepwise approach to satisfy both conditions.

The design of EQUIPT has several strengths. The European roll-out of the ROI tool is based on prior research in the UK. The 'inverted cone' approach to EQUIPT allows us to test the transferability of economic evidence in a logical pathway. This approach is likely to avoid any noise in drawing policy implications from the study results. Stakeholder engagement throughout the research process highlights the design to be highly relevant to end-users of research findings.

EQUIPT is not free of challenges, however. The country-specific modelling process will require most relevant country-specific data, which are often scarce. A related limitation concerns the availability of effectiveness data on the full-range activities and strategies recommended by, for example, the WHO, the US Surgeon General's report and the Centre for Disease Control. To aid in the transfer of evidence where such data are not available, the project is set up to seek to identify those input data that cause the most variability with respect to outcomes. In addition, the selection of the four sample countries is designed to provide a wide representation of the smoking cessation context within Europe, thereby aiding in the adaptation of the model to inform policy within additional countries.

In the worst-case scenario of extreme scarcity of relevant data in a country, EQUIPT will seek an expert panel to make decisions on the best available evidence for such a case. By doing so, EQUIPT will highlight the gaps where more research needs to be conducted and/or more data need to be collected. Furthermore, while the transferability of study results to out-of-sample countries is a complex endeavour, this needs to be communicated to end-users in simple, practical and customisable webbased tools. Thanks to the 'inverted cone' approach, the stakeholder engagement as well as modelling experience gathered in sample countries will inform us to mitigate such challenges. 
Author affiliations

${ }^{1}$ Health Economics Research Group, Institute of Environment, Health and Societies, Brunel University London, London, UK

${ }^{2}$ Maastricht University, Caphri School of Public Health and Primary Care, Maastricht, The Netherlands

${ }^{3}$ Trimbos Institute, Netherlands Institute of Mental Health and Addiction, Utrecht, The Netherlands

${ }^{4}$ Institute of Health Economics and Health Care Management, Helmholtz Zentrum München, German Research Center for Environmental Health, Member of the German Center for Lung Research (DZL), Munich, Germany ${ }^{5}$ Universitat Pompeu Fabra, Barcelona, Spain

${ }^{6}$ Faculty of Social Sciences, Department of Health Policy and Health Economics, Eötvös Loránd University, and Syreon Research Institute, Budapest, Hungary

${ }^{7}$ Maastricht University, Caphri School of Public Health and Primary Care, Maastricht, The Netherlands

${ }^{8}$ Tobacco Free Futures, Manchester, UK

${ }^{9}$ Smokefree South West, Bristol, UK

${ }^{10}$ FRESH Smokefree North East, Durham, UK

${ }^{11}$ LeLan (Ltd) Solutions, Bristol, UK

${ }^{12}$ Department of Epidemiology and Public Health, Health Behaviour Research Centre, University College London, London, UK

${ }^{13}$ National Centre for Smoking Cessation and Training, London, UK

${ }^{14}$ National Institute for Health and Care Excellence, London, UK

${ }^{15}$ European Network for Smoking Prevention, Brussels, Belgium

${ }^{16}$ Department for Development, Research and Health Technology Assessment,

Agency for Quality and Accreditation in Health Care and Social Welfare,

Zagreb, Croatia

${ }^{17}$ Department of Epidemiology \& Community Medicine, Faculty of Medicine,

University of Ottawa, Ottawa, Canada

Contributors All authors conceived the study, participated in a proposal development workshop and subsequently applied for funding. SP wrote the first draft of the manuscript with support from DC, KC, AL-G and MV and was responsible for the final editing. DC, KC, SE, RL, MT-B and LO provided critical inputs to the economic modelling component; $A C, F A$ and $A R$ contributed to policy component; RW, TJ and ZV to interventions and effectiveness components; HdV, AL-G and CR-L to dissemination component; and $\mathrm{ZK}, \mathrm{MH}$ and $\mathrm{SP}$ to international transferability component. All authors have read and approved the final manuscript. SP is the guarantor.

Funding We have received funding from the European Community's Seventh Framework Programme under grant agreement No. 602270 (EQUIPT).

Competing interests RW undertakes consultancy and research for and receives travel funds and hospitality from manufacturers of smoking cessation medications but does not, and will not take funds from e-cigarette manufacturers or the tobacco industry. RW is an honorary co-director of the National Centre for Smoking Cessation and Training and a Trustee of the stop-smoking charity, QUIT. RW's salary is funded by Cancer Research UK.

Ethics approval Brunel University Research Ethics Committee.

Provenance and peer review Not commissioned; internally peer reviewed.

Data sharing statement The relevant data will be available to download from the EQUIPT website (http://equipt.ensp.org). This will include a list of model parameters and their values.

Open Access This is an Open Access article distributed in accordance with the Creative Commons Attribution Non Commercial (CC BY-NC 4.0) license, which permits others to distribute, remix, adapt, build upon this work noncommercially, and license their derivative works on different terms, provided the original work is properly cited and the use is non-commercial. See: http:// creativecommons.org/licenses/by-nc/4.0/

\section{REFERENCES}

1. European Commission. Public health: tobacco policy. European Commission, 2012
2. Ross $\mathrm{H}$. The economics of tobacco and tobacco control in the European Union. In: McNeill A, Godfrey F, Koehn W, eds. Tobacco or health in the European Union: past, present and future. Brussels: The ASPECT Consortium, European Commission, 2004.

3. WHO. WHO Framework Convention on Tobacco Control. WHO, 2003.

4. Joossens L, Raw M. The Tobacco Control Scale: a new scale to measure country activity. Tob Control 2006;15:247-53.

5. Cromwell J, Bartosch WJ, Fiore MC, et al. Cost-effectiveness of the clinical practice recommendations in the AHCPR guideline for smoking cessation. JAMA 1997;278:1759-66.

6. NICE. Smoking and tobacco (guidance). National Institute for Health and Care Excellence, 2012

7. NICE. Supporting investment in public health: review of methods for assessing cost-effectiveness, cost impact and return on investment. Proof of concept report. National Institute for Health and Care Excellence, 2011.

8. Pokhrel S, Owen L, Lester-George A, et al. Tobacco control return on investment tool. London: National Institute for Health and Care Excellence, 2014

9. Boehler C. Mind the gap! Geographic transferability of economic evaluations in health. Brunel University, 2013.

10. Drummond $\mathrm{M}$, Barbieri $\mathrm{M}$, Cook J, et al. Transferability of economic evaluations across jurisdictions: ISPOR Good Research Practices Task Force report. Value Health 2009;12:409-18.

11. Goeree R, Burke N, O'Reilly D, et al. Transferability of economic evaluations: approaches and factors to consider when using results from one geographic area for another. Curr Med Res Opin 2007;23:671-82.

12. Kalo Z, Landa K, Dolezal T, et al. Transferability of National Institute for Health and Clinical Excellence recommendations for pharmaceutical therapies in oncology to Central-Eastern European countries. Eur J Cancer Care (Engl) 2012;21:442-9.

13. Trapero-Bertran M, Pokhrel S, Trueman P. An economic model of tobacco control version 1. Tobacco Free Futures, FRESH Smokefree North East \& Smokefree South West. Health Economics Research Group, Brunel University, 2011.

14. Rogers EM. Diffusion of innovations. 5th edn. New York: Free Press, 2003.

15. Ogilvie $D$, Craig $P$, Griffin $S$, et al. A translational framework for public health research. BMC Public Health 2009;9:116.

16. WellcomeTrust. What is translational research? WellcomeTrust, 2012.

17. Vries HD, Mesters I, Steeg HVD, et al. The general public's information needs and perceptions regarding hereditary cancer: an application of the Integrated Change Model. Patient Educ Couns 2005;56:154-65.

18. Flack S, Taylor M, Trueman P. Cost-effectiveness of interventions for smoking cessation. Final Report 2007a. National Institute for Health and Care Excellence, 2007.

19. Weinstein MC, O'Brien B, Hornberger J, et al. Principles of good practice for decision analytic modeling in health-care evaluation: report of the ISPOR task force on good research practicesmodeling studies. Value Health 2003;6:9-17.

20. Drummond M, O'Brien B, Sculpher M, et al. Methods for the economic evaluation of health care programmes. 3rd revised edn. Oxford: Oxford University Press, 2005.

21. Philips Z, Bojke L, Sculpher M, et al. Good practice guidelines for decision-analytic modelling in health technology assessment: a review and consolidation of quality assessment. Pharmacoeconomics 2006;24:355-71.

22. NICE. Methods for the development of NICE public health guidance. 3rd edn. London: National Institute for Health and Care Excellence, 2012.

23. EUnetHTA. HTA core model for medical and surgical interventionsWork Package 4-The HTA Core Model. v. 1.0. European Network for Health Technology Assessment, 2008.

24. Drummond MF, Jefferson TO. Guidelines for authors and peer reviewers of economic submissions to the BMJ. BMJ 1996:313:275-83.

25. Welte $\mathrm{R}$, Feenstra $\mathrm{T}$, Jager $\mathrm{H}$, et al. A decision chart for assessing and improving the transferability of economic evaluation results between countries. Pharmacoeconomics 2004;22:857-6.

26. Coyle D, Buxton MJ, O'Brien BJ. Measures of importance for economic analysis based on decision modeling. J Clin Epidemiol 2003:56:989-7.

27. Sculpher MJ, Pang FS, Manca A, et al. Generalisability in economic evaluation studies in healthcare: a review and case studies. Health Technol Assess 2004;8:iii-iv, 1-192. 